\title{
Effect of 6 Weeks of Aerobic Training with Nanocurcumin Consumption on IL1 $\beta$, Nitric Oxide, and Depression in Women with Metabolic Syndrome
}

\author{
Ali Osali ( $\square$ osalialiphd@gmail.com ) \\ University of Bonab https://orcid.org/0000-0002-3924-2045 \\ Alireza Rostami \\ University of Bonab
}

Research article

Keywords: Aerobic exercise, inflammation, depression, metabolic syndrome, nanocurcumin

Posted Date: April 22nd, 2021

DOl: https://doi.org/10.21203/rs.3.rs-429806/v1

License: (c) (1) This work is licensed under a Creative Commons Attribution 4.0 International License.

Read Full License 


\section{Abstract}

Backgrounds and Objectives

The use of anti-inflammatory supplements is important in improving the executive function of obese people. The aim of this research was to investigate the effect of 6 weeks of aerobic exercise with moderate intensity and consumption of nanocurcumin on IL1 $\beta$, nitric oxide, and depression in women aged 60-65 with metabolic syndrome.

Materials and Methods

44 women with metabolic syndrome (MetS) were randomly selected and divided into four groups of 10, including MetS exercise+ Curcumin (MECU), MetS exercise (ME), MetS NanoCurcumin (MCU), MetS control (MC Training groups performed an exercise protocol of aerobic exercise for 6 weeks (three sessions per week). Blood samples were obtained before and after the training period for antioxidant indicators and lipid degradation measurement. Also, the Beck anxiety questionnaire was used for evaluating levels of anxiety. T-test and one-way analysis of variance were used for the evaluation of within-group and between-group differences, respectively.

Results

There was a significant difference in IL1 $\beta$, nitric oxide (NO), and depression before and after exercise in all three experimental groups $(p \leq 0.05)$. Also, the results showed a significant difference in the level of NO and depression in the research groups. The highest decrease in these variables was observed in the aerobic training group and training with nanocurcumin consumption group $(p \leq 0.05)$.

Conclusion

These findings indicated that six-week nanocurcumin supplementation with aerobic training is a suitable method for reducing IL1 $\beta$ and NO and depression, preventing metabolic, cardiovascular, and inflammatory diseases in women with metabolic syndrome.

Name of the registry: IR.SEMUMS.REC.1396.107

Trial registration number: IRCT2017082335857N1

\section{Introduction}

Aging is an inevitable fact, The increase in the elderly population around the world is an important concern, The World Health Organization estimated the total number of elderly people in the world in 2006 at about 700 million and it will double in the next 40 years, In Iran, the population over the age of 60 will reach about 10 million by 2020 and more than 26 million by 2050 (1). Depending on the social and family circumstances of the elderly, 12 to $16 \%$ of the elderly are likely to be depressed (1). Depression 
reduces the quality of life of the elderly and increases their dependence on others (1). Depression increases drug use, length of hospital stay, and care costs (1). Its prevalence is also expected to increase to more than $15-20 \%(1,2)$. Symptoms of depression: include sadness, irritability, decreased interest in fun activities, significant weight gain and loss, insomnia or excessive sleep, decreased memory, decreased libido, fatigue, loss of energy, worldly worthlessness, excessive guilt, decreased power of concentration, and increased suicidal ideation (2-4). Biologically, depression is a motivational disorder caused by a lack of biogenic amino acids. Biogenic amines are neurochemicals that facilitate neurotransmission and play an important role in the pathology of mood disorders. Speculation about the neurochemical basis of depression is often about reduced serotonin and norepinephrine (5). Serotonin levels are related to the amount of tryptophan in the blood. Researchers have shown that $10 \%$ of tryptophan is freely present in the blood, with free tryptophan entering the brain and the rest binding to albumins. The binding site of tryptophan and fatty acids to albumin is the same. Some researchers have shown that long-term aerobic exercise increases the recall of fatty acids, and therefore the amount of free tryptophan in the blood increases due to the competitive binding of fatty acids and tryptophan to albumin. Therefore, the rate of serotonin synthesis increases in the brain (6-8). Curcumin was used in China and India to treat some diseases (9). Fundamental studies on the medicinal properties of curcumin have shown its effect on lowering blood sugar (10-12), anti-inflammation - (2), and antioxidant properties (13) of turmeric (2). Aging and metabolic syndrome are associated with increased chronic inflammation (1). Due to lifestyle changes, the prevalence of metabolic syndrome has increased in today's society (1). Metabolic syndrome refers to the presence of at least 3 of 5 risk factors. These factors include: waist fat of greater than $94 \mathrm{~cm}$, triglyceride of higher than 150, HDL level of less than 40, hyperglycemia (glucose higher than 110), and blood pressure of higher than $85 / 130$ (1). Some researchers have suggested that a decrease in serotonin results in an increase in inflammation $(14,15)$. The exercise and curcumin supplements both reduce inflammation, and exercise and curcumin supplementation can be used as a treatment strategy to improve severe depression $(5,16-18)$. In this regard, research has been done, which will be briefly overviewed. Osali et al. (2017) expressed the positive effect of exercise and curcumin consumption on reducing inflammatory factors and depression. Kulkarni et al. (2008) reported curcumin supplementation as effective in reducing depression (16). Legrand et al. (2009) showed the effect of regular walking on reducing depressive symptoms in women (19). Mokhtari et al. (2013) also reported the effect of 12 weeks of aerobic exercise on reducing depression (20). Dey et al. (1994) stated that 4 weeks of moderate-intensity swimming is effective in improving the behavior of depressed people (21). Donohue et al. (2004) also reported no effect of exercise training on depression in young athletes (22).

Nitric oxide (NO) has multiple biological actions and can regulate physiology acutely or lead to long-term changes in cell function. The pleiotropic roles of $\mathrm{NO}$ include the regulation of long-term synaptic transmission, learning, memory, platelet aggregation, leukocyte-endothelial interactions, immune function, angiogenesis, and arteriogenesis. However, NO is generally well-known as a potent regulator of blood flow and was originally termed endothelial-derived relaxing factor (EDRF). The story was unfolded from Furchgott and Zawadzki's initial discovery that endothelial cells control acetylcholine-induced relaxation of smooth muscle muscles. A few years later, NO was identified as the key endothelium-derived molecule 
promoting vasodilation: NO synthesized by NOS in the endothelium diffuses into the vessel wall where it activates sGC in the vascular smooth muscle; this leads to a rise in cyclic GMP (cGMP) and elicits relaxation of the vessel. However, it readily became apparent that different isoforms of NOS have different physiological functions. For example, NOS3 and NOS1 were found to have distinct roles in regulating microvascular tone; Overall, NO derived from the integration of NOS3 and NOS1 activities plays a key role in regulating systemic blood pressure and acutely regulating organ blood flow, whereas NOS2-derived NO species are most well recognized for their impact on pathogen killing and inflammatory processes (23).

The absorption of curcumin in turmeric is very low due to its lipophilic nature. In fact, there is a layer of water on the surface of intestinal epithelial cells. For a drug to be absorbed from the intestine after oral administration, it must have minimum solubility in water to pass through this layer after dissolving in water. In other words, small amounts of curcumin in the gut (such as lipophilic substances) are converted to mesylate and absorbed. The curcumin capsules contain nanocurcumin with a size of $10 \mathrm{~nm}$, and its solubility in water is 100 times more than that of turmeric powder, and this factor causes it to absorb. After oral consumption of soft gel capsules which contain nanocurcumin, it opens in less than 15 minutes in acidic gastric medium and distributes in all of gastric. These nanocurcumins in acidic gastric conditions are constant at least 6 hours, and they change the intestine intake manner. With regard to the increasing curcumin absorption when using it as a nano drug, the question raised is that the 80 milligram consumption of nanocurcumin cause a significant decrease in depression, NO, and IL1 $\beta$ ?"

\section{Methodology}

The method of quasi-experimental study is applied studies. The research design included the pre-test and post-test with one control group and three experimental groups All stages of the research were approved by the ethics committee of Semnan University of Medical Sciences with the license number IR.SEMUMS.REC.1396.107 Also, this research has been registered in the Clinical Trial Registration Center of Iran under number IRCT2017082335857N1.

The statistical population of the present study was 250 wives of martyrs aged 50 to 65 in Zanjan. Sampling was purposeful. After distributing an advertisement in the General Office of the Martyr and Veterans Affairs Foundation of Zanjan Province and sending an invitation to the statistical community, at the beginning of the research, 65 females volunteered to to participate in in the study. They were examined by a physician for their history of illness and physical distress, psychological problems, sleep, and blood pressure, and if necessary, some of them underwent heart-health tests. None of the subjects had a history of regular physical activity in the past year. It should be noted that in this study, the ATPIII (Adult treatment panel iii) criterion was used to identify metabolic risk indicators, which were considered in the presence of three of the five indicators (waist circumference more than $94 \mathrm{~cm}$, blood triglyceride more than $150 \mathrm{mg} / \mathrm{dL}$, blood HDL less than $40 \mathrm{mg} / \mathrm{dL}$, blood pressure more than $130.85 \mathrm{~mm} \mathrm{Hg}$ and fasting blood glucose higher than $110 \mathrm{mg} / \mathrm{dL}$ ). In other words, volunteers with three or more metabolic risk indices based on ATPIII criteria were considered as subjects with metabolic syndrome (1). 


\section{Training protocol and supplementation}

The subjects were allowed to practice from 9 a.m. to 12 p.m. The training period was six weeks. They practiced 3 days a week. Each session, the exercises were performed in the form of three consecutive sets with a break interval of 5 minutes between sets. The training set time in the first week was 12 minutes, and with the passing of each week, one minute was added to the duration of the training sets, so that in the sixth week of the training, it reached three sets of 17 minutes. The exercise was performed at an intensity of 65 to $75 \%$ of the heart rate reserve. Resting heart rate was checked weekly, and the intensity of the exercise program was adjusted using a Polar (Finland) pacemaker. The whole training session started with 5 minutes of warming up (flexibility and stretching exercises) and ended with 5 minutes of cooling down. The control group avoided, regular physical activity during this six-week period. The stored heart rate was calculated using the Carvonen formula (1).

60 to $70 \%$ reserve heart rate= [(Maximum heart rate - Resting heart rate $(60$ to $70 \%)]+$ Resting heart rate Heart rate when waking up and lying down before getting out of bed = resting heart rate

\section{Amount and method of receiving nanocurcumin}

The subjects received an $80 \mathrm{mg}$ of nanocurcumin tablet each morning.

\section{Measurement of biochemical parameters}

All subjects underwent fasting blood sampling at 9 am (to measure plasma levels of IL $1 \beta$ and serum glucose, triglyceride, and plasma doped lipoprotein) in two stages, including the pre-test and post-test (after six weeks). In order to eliminate the acute effects of exercise such as delayed contusion and small possible injuries to the muscle structure on the serum level of $I L 1 \beta$, blood sampling was performed in the post-test phase four days after the last training session $(23,24)$. At each blood sampling, blood vessels of the brachial vein were collected in tubes without EDTA anticoagulant. After centrifugation (12 minutes at $3000 \mathrm{rpm}$ ) and separation of serum, serum IL1 $\beta$ levels were measured by ELISA, a special kit for measuring IL1 $\beta$ (eBioscience, Vienna, Austria) with a sensitivity of $0.05 \mathrm{pg} / \mathrm{mL}$. Blood glucose was measured by glucose oxidase, and fat levels were measured by a standard enzymatic method (Pars Kit test, Karaj, Iran) using Kubas Mira biochemical AutoAnalyzer. The coefficient of variation of this kit in each assay and between different assays (inter-assay variation) was equal to $1.82 \%$ and $1.6 \%$ for triglycerides, $1.74 \%$ and $1.19 \%$ for blood sugar, and $2.15 \%$ and $1.28 \%$ for $\mathrm{HDL}$, respectively. (NO) is extremely unstable and undergoes rapid oxidative degradation to stable nitrite (NO2-) and nitrate (NO3-), which react with the colorant and produce azo-pink composition, and it is quantified spectrophotometrically (27). Serum levels of metabolites were measured by colorimetric Griess assay. During the colorimetric assay, the nitrite concentration was determined by measuring the absorbance at $450 \mathrm{~nm}$. 
The Beck Depression Inventory (BDI) is a 21-item, self-rated scale that evaluates key symptoms of depression, including mood, pessimism, sense of failure, self-dissatisfaction, guilt, punishment, selfdislike, self-accusation, suicidal ideas, crying, irritability, social withdrawal, indecisiveness, body image change, work difficulty, insomnia, fatigability, loss of appetite, weight loss, somatic preoccupation, and loss of libido (26).

\section{The calculation of body fat percentage}

The percentage of body fat of the subjects was calculated by the pneumatic composite device, model OMRON BF500 made in Germany.

\section{The calculation of calorie intake}

Subjects recorded their daily food intake in the notebook before starting the training protocol (beginning, middle, and end of the week), and then the calories of food consumed for breakfast, snacks, lunch, and dinner were calculated by N4 software. The results showed no significant difference in the number of calories consumed between the groups (1).

\section{Statistical analysis}

Data were presented as mean \pm SD. Levene's test was used to test the homogeneity of variances in the pre-test. The Kolmogorov-Smirnov test was used to ensure that the distribution of the variables was normal. After verifying the normality of data, to analyze the data,paired sample t-test was used to compare the pre-test and post-test means within the group, and the one-way analysis of variance test (ANOVA) (difference between test and post-test) was used to compare the groups. Also, in the case of significant findings, Bonferroni's post hoc test was used. All data analysis was performed using SPSS software version 24 , the significance level was set at 0.05 .

\section{Results}

The physical qualities of subjects were measured twice: at the first and the last sessions. The measures are as follows in the last session: (expressed as mean \pm standard deviation [SD]): age, $62.3 \pm 1.23$ years; height, $164 \pm 7 \mathrm{~cm}$; body weight, $81.2 \pm 2.4 \mathrm{~kg}$; and body mass index (BMI), $29.5 \pm 1.2 \mathrm{~kg} / \mathrm{m}^{2}$. The results of group comparison of metabolic syndrome, weight, fat percentage, BMI, IL1 $13, \mathrm{NO}$, and depression are listed in Tables 1 and 2. The results of paired sample t-test showed that IL1 $\beta$ levels in the training group $(p=0.003)$, nanocurcumin consumption $(p=0.05)$, and training with nanocurcumin $(p=0.002)$ decreased significantly in posttest compared to pretest. IL $1 \beta$ did not change significantly in the post-test of the control group compared to the pre-test time $(p=0.8)$. Also, NO levels in the training group $(p=0.002)$, nanocurcumin consumption ( $p=0.002)$, and training with nanocurcumin consumption $(p=0.002)$, decreased significantly in the posttest compared to the pretest. NO did not change significantly in the posttest in comparison with the pre-test in the control group $(\mathrm{p}=0.5)$ (Table 2). The results of the one-way ANOVA showed that levels of IL1 $\beta$ in the training, nanocurcumin, and training with nanocurcumin groups 
were significantly lower than the control group $(p=0.002)$ (Table 2$)$. The results of the Bonferroni post-hoc test of IL1 $\beta$ showed no difference between training and training with nanocurcumin groups $(p=0.1)$, but there was a difference between nanocurcumin and training groups $(p=0.000)$, training and control groups $(p=0.000)$, training with nanocurcumin and nanocurcumin groups $(p=0.002)$, nanocurcumin and control groups $(p=0.000)$, and training with nanocurcumin and control groups $(p=0.002)$. Also, levels of NO in training, nanocurcumin, and training with nanocurcumin groups were significantly lower than that of the control group ( $p=0.002)$. The results of Bonferroni post hoc NO test showed no difference between training and nanocurcumin groups $(p=0.1)$. But differences were shown between training and training with nanocurcumin groups $(p=0.006)$, training and control groups $(p=0.02)$, nanocurcumin and training with nanocurcumin groups $(p=0.001)$, nanocurcumin and control groups $(p=0.007)$, training with nanocurcumin and control groups $(p=0.002)$. Also, depression in the exercise + supplement group was higher than that for the exercise and supplement groups, and this difference was statistically significant.

\section{Discussion}

The reduction in depression and IL1 $\beta$ and NO was significant after six weeks of aerobic exercise and nanocurcumin use. Reducing depression is consistent with the results of Dimeo et al. (2001), Hematfar et al. (2012), HekmatiPour et al. (2013), Mokhtari et al. (2013), and Osali et al. (2017) $(1,5,18,20,25)$. HekmatiPour et al reported the effect of 4 weeks of training, 4 sessions per week for 25 minutes, on reducing depression in the elderly (26). Hematfar et al reported 8 weeks of aerobic exercise as effective in reducing depression and increasing serotonin in students aged 25-18 years (5). Mokhtari et al. also reported 12 weeks of aerobic exercise to be effective in improving balance and significantly reducing depression in older women aged 80-62 (20). Osali et al. reported that three months of moderate-intensity aerobic exercise was effective in reducing depression and IL- 6 in elderly women with metabolic syndrome (1). In another study, Osali (2017) investigated the effect of eight weeks of turmeric consumption and aerobic exercise with an intensity of $75-65 \%$ of stored heart rate on IL-6 plasma levels and depression in elderly women with metabolic syndrome. The results reported by Osali (2017) is similar to the results of the present study. The same result was obtained when the amount and duration of curcumin use in the present study were less than what used in the previous study (2017). Curcumin used in the present study was in the form of $80 \mathrm{mg}$ nanocurcumin tablets, but in the previous study (2017), the daily consumption of curcumin was $20 \mathrm{mg}$ per $\mathrm{kg}$ of body weight. The reduction of depression in this study is not consistent with the results of Donohue et al. (2004) (22). Donohue et al. (2004) showed that young people who exercised regularly were more likely than other non-athlete youths to have impulsive, neurotic, and psychotic disorders. Considering the age of the subjects and the purpose of the exercise in this age, it can be said that the attitude towards exercise is effective in reducing and increasing depression and behavioral disorders. In the present study, exercise was performed to treat and reduce depression, which showed a significant reduction in blood pressure, body fat percentage, weight, waist size, and triglyceride. But in the study by Donohue et al. (2004), exercise was aimed at achieving results (championships), which increases behavioral disorders. Differences in the age of subjects, body composition, and purpose of the exercise are among the reasons for inconsistent results. The mechanism of the effect of exercise 
and nanocurcumin consumption on reducing depression in women aged 65-60 with metabolic syndrome can be investigated through several pathways. The first pathway is the effect of exercise and nanocurcumin consumption on IL1 $\beta$ reduction. In the present study, the decrease in IL1 $\beta$ can be attributed to a significant decrease in blood pressure, triglyceride, and waist size as well as a significant increase in HDL. This is consistent with Zorilla et al. (2001) and Mossner et al. (2007) who pointed out that the increase in inflammatory factors is associated with a decrease in serotonin $(14,15)$. The reduced depression can be attributed to a decrease in IL1 $\beta$, and a decrease in IL $1 \beta$ is effective in increasing serotonin (17).

The second pathway is the effect of exercise on increasing tryptophan levels. Tryptophan is a precursor to the synthesis of serotonin, which is increased by prolonged aerobic exercise. The mechanism of an increase in tryptophan: involves the transportation of fatty acids and tryptophan in the blood by albumin, both of which have the same binding site on albumin, While training, the amount of fatty acid increases, and this increase leads to a decrease in the albumin site for the binding of tryptophan to albumin. Increased free tryptophan in the blood leads to more tryptophan entering the brain, and the presence of tryptophan in the central nervous system leads to more synthesis of serotonin and a relief from depression (6-8). Curcumin also inhibits both monoamine oxidase $A$ and $B$ activity. Monoamine oxidases $A$ and $B$ are oxidizers of dopamine, norepinephrine, and serotonin, whose oxidation is reduced by curcumin and consequently the levels of dopamine, norepinephrine, and serotonin is reduced $(2,16,27)$. Previous studies concerning serum or plasma cytokine levels and their concentrations have evaluated the acute effects of exercise. Based on the findings reported in previous as well as the present study, it could be hypothesized that major weight loss connected to exercise training is necessary to modulate inflammatory indicators levels. Also, we found a significant reduction of IL- 6 and CRP in women with metabolic syndrome. In this regard, some of the previous studies have shown significant reductions in Creactive protein concentration which is another important indicator of systemic inflammation, following nano-curcumin intake and supplementation (28). Increased expression of inflammatory factors causes the activity of nuclear factor kappa B (NF-KB). NF-KB binds to DNA to block the expression of BDNF (brain-derived neurotrophic factor). Curcumin reduces IL1 $\beta$ expression and NF-KB transcription, which in turn increases BDNF expression $(2,17)$. BDNF is a neurotrophic factor that causes both neurogenesis and neuroplasticity (28-30). Due to the fact that neurotransmission is biologically impaired in depressed people (5), significant decrease in waist circumference, blood pressure, and triglyceride as well as an increase in HDL due to two months of moderate-intensity aerobic exercise significantly reduced IL1 $\beta$ and decreased NF-KB activity and binding to DNA. This process leads to increased BDNF expression, and increased BDNF also improves neurotransmission and relieves depression $(2,31)$. The present study showed that doing exercises for 6 weeks with nanocurcumin supplementation had a significant effect on NO levels in women aged 60-65 with metabolic syndrome. Chematic of changes in NOS3 or NO: involves the following ones (A) Decreased NOS3 expression commonly occurs in obese and diabetic states. The mechanisms proposed for diminished expression include TNF-a-mediated destabilization of NOS3 mRNA, which may involve eEF1A1. High levels of NO may regulate NOS3 abundance through cGMPmediated or via NF-KB-SNO feedback regulatory pathways. A small 27-nt RNA regulates NOS3 expression, 
nevertheless, it is not known whether this mechanism is invoked in obesity or diabetes. (B) Decreased NOS3 activity in obesity and diabetes is largely attributed to insulin resistance, which may be mediated by free fatty acid (FFA)-induced activation of TLR2, TLR4, and NF-KB. In addition, activation of PKC $\beta$ II may diminish Akt signaling, which normally promotes phosphorylation of NOS3 on Ser1177.

Phosphorylation at this site increases NO output by the enzyme. Hyperglycemia may also lead to increased O-GIcNAcylation of NOS3, which decreases Ser1177 phosphorylation and inhibits its activity. In addition, conditions leading to obesity promote upregulation of Cav-1, which is a negative regulator of NOS3, and ceramide accumulation disrupts the NOS3-Akt-HSP90 complex, diminishing the activity of the enzyme. (C) NOS3 may also be uncoupled or NO quenched in obese and diabetic states. Diminished levels of substrates and cofactors, such as L-arginine or tetrahydrobiopterin (BH4), lead to uncoupling of the enzyme, which is commonly associated with the presence of NOS3 monomers rather than dimers and can produce superoxide instead of NO. Endogenous inhibitors of NOS3 such as ADMA are also increased in obese conditions and can promote NOS uncoupling. Elevated production of reactive oxygen species such as superoxide can quench $\mathrm{NO}$ and result in its oxidation to highly reactive peroxynitrite, which damages biomolecules and can oxidize $\mathrm{BH} 4$ to $\mathrm{BH} 2$ (23).

\section{Conclusion}

Due to a decrease in IL $1 \beta$, NO, and depression, the improvement of metabolic syndrome was made following six weeks of moderate-intensity training and nanocurcumin consumption, Reduced depression can be attributed to these factors. This study thus suggests aerobic exercise with nanocurcumin use as a non-invasive strategy in the treatment of depression in women aged 60-65 with metabolic syndrome.

Finally, it is suggested to conduct research with different intensity and duration of training on the target community.

\section{Abbreviations}

MetS: Metabolic syndrome

SBP: systolic blood pressure

BMI: Body mass index

WHR: waist hip ratio

NO: Nitric oxide

IL1ß: Interleukin 1 beta

\section{Declarations}

Ethics approval and consent to participate 
University of Bonab Review Board for the protection of human subjects (Approval date, 2018-July-17) approved this study.

\section{Consent for publication}

All subjects were aware during the informed consent process that the results of this study may be published.

\section{Availability of data and materials}

Not applicable. Conclusions of the manuscript are based on relevant data sets available in the manuscript.

\section{Competing interests}

The authors declare that they have no competing interests.

\section{Funding}

This article is taken from research project number 97/I/ER/1920, sponsored by University of Bonab, Iran.

\section{Authors' contributions}

The study was designed by $\mathrm{OA}$; data were collected and analyzed by $\mathrm{OA}$; data interpretation and manuscript preparation were undertaken by $A R$.

\section{Acknowledgements}

We thank to all the subjects who voluntarily participated, and we also gratefully thank dear president of Martyr Foundation and Sacrifice Affairs who sincerely helped us in conducting this research.

\section{Affiliations}

Exercise physiology, physical education and sport sciences, University of B, B, I

Corresponding Author: A O, Department General Courses, University of B, B, I

E-mail: osalialiphd@gmail.com

\section{References}

1. Osali A, Mostafavi H, Moaseri F. The Effect of Twelve-Week Aerobic Exercise on IL-6 and depression in 50-65 Years Old Women with Syndrome Metabolic. TUOMS. 2017.

2. Kulkarni S.K, Dhir Ashish, Akula Kiran Kumar. Potentials of Curcumin as an Antidepressant. SWJ. 2009;9:1233-41. 
3. Ballard C, Bannister C, Solis M, Oyebode F, Wilcock G. The prevalence, associations and symptoms of depression amongst dementia sufferers. J Affect Disord. 1996;36:135-44.

4. Stepaniuk J, Ritchi L, Tuokko H.A. Neuropsychiatric impairments as predictors of mild cognitive impairment, dementia, and Alzheimer's disease. Am J Alzheimers Dis. 2008;23:326-33.

5. Hematfar A, shahsavari A, Tip H. The Effect of Eight Weeks of Selected Aerobic Exercise on the Depression and Serum Serotonin Concentration in Depressed Female University Students. journal of sport biosciences. 2012;4(13):51-62.

6. Osali A, Kordi M, Azad A. The Effects of Carbohydrates and Branched Chain Amino Acid Supplements Consumption in Recovery Period on Secretion of Insulin and Preserving the Performance of Wrestlers. journal of sport biosciences. 2009;1(2):129-272.

7. Davis JM, S.P. Bailey, J.A.Woods, F.J. Galiano, M.T. Hamilton, Bartoli WP. Effects of carbohydrate feedings on plasma free tryptophan and branched-chain amino acids during prolonged cycling. . European Journal of applied and Occupational Physiology. 1992;65:513-9.

8. Blomstrand E. Amino acids and central fatigue. Amino Acids J Physiol Endocrinol Metab. 2001;20(1):25-34.

9. Aggarwal B.B, Sundaram C, Malani N, Ichikawa H. Curcumin: the Indian solid gold. Adv Exp Med Biol. 2007;595:1-75.

10. Srinivasan M. Effect of curcumin on blood sugar as seen in a diabetic subject. Indian J Med Sci. 1972;26:269-70.

11. Babu P.S, Srinivasan K. Influence of dietary curcumin and cholesterol on the progression of experimentally induced diabetes in albino rat. Mol Cell Biochem. 1995;152:13-21.

12. Arun N, Nalini N. Efficacy of turmeric on blood sugar and polyol pathway in diabetic albino rats. Plant Foods Hum. Nutr. 2002;57:41-52.

13. Sreejayan R, Rao M.N. Curcuminoids as potent inhibitors of lipid peroxidation. J Pharm Pharmacol. 1994;46:1013-6.

14. Zorilla EP LL, McKay JR, Roesnthal R, Houldin A, Tax A, et al. . The relationship of depression and stressors to immunological assays: A meta-analytic review. Brain Behav Immun. 2001;15:199-226.

15. Mossner R MO, Koutsilieri E, Saoud M, Ehlis AC, Muller N, et al. Consensus paper of the WFSBP Task Force on Biological Markers: Biological markers in depression. World J Biol Psychiatry. 2007;8:14174.

16. Kulkarni S.K, Bhutani M.K, Bishnoi M. Antidepressant activity of curcumin: involvement of serotonin and dopamine system. Psychopharmacology. 2008;201:435-42.

17. Lim G.P, Chu T, Yang F, Beech W, Frautschy S.A, Cole G.M. The curry spice curcumin reduces oxidative damage and amyloid pathology in an Alzheimer transgenic mouse. Neurosci. 2001;21:8370-7.

18. Osali A. The Effect of Eight -Week Aerobic Exercise and consumption of curcumin on IL1 $\beta$ and depression in 60-65 Years Old Women with Syndrome Metabolic. TUOMS. 2017. 
19. Legrand F, Mille $\mathrm{CH}$. The effects of 60 minutes of supervised weekly walking (in a single vs.3-5 session format) on depressive symptoms among older women: Findings from a pilot randomized trial. Mental Health and Physical Activity. 2009;2:71-5.

20. Mokhtari M, Nezakatalhossaini M, Esfarjani F. The effect of 12-week pilates exercises on depression and balance associated with falling in the elderly. Procedia - Social and Behavioral Sciences. 2013(70):1714-23.

21. Dey S. Physical exercise as a novelantidepressant.agent: Possible role of serotonin receptor subtypes. Phsiology\&Behavior. 1994;55(2):323-9.

22. Donohue B. Examination of Psychiatric Symptom In Student Athletes. Journal Of Gen Psycho. 2004;131(1):29-36.

23. Pacher P, Beckman JS, Liaudet L. Nitric oxide and peroxynitrite in health and disease. Physiol Rev. 2007;87:315-424.

24. Patanella AK, Zinno M, Quaranta D, Nociti V, Frisullo G, Gainotti G, et al. Correlations Between Peripheral Blood Mononuclear Cell Production of BDNF, TNF-alpha, IL-6, IL-10 and Cognitive Performances in Multiple Sclerosis Patients. Journal of Neuroscience Research. 2010;88:1106-12.

25. Qi Z, He J, Zhang Y, Shao Y, Ding S. Exercise training attenuates oxidative stress and decreases p53 protein content in skeletal muscle of type 2 diabetic Goto-Kakizaki rats. Free Radic Biol Med. 2011; $50(7): 794-800$.

26. Dimeo F, Bauer M, Varahram I, Proest G, Halter U. Benefits from aerobic exercise in patiwnts with major depression: a pilot study. The British journal of Sports Medicine. 2001;35:114-7.

27. Nafiseh HekmatiPour, Hamid Hojjati, Hamid Sharif Nia, Golbahar Akhondzade, Alireza Nikjou, Mansoureh MirAbolhasani. Impact of Routine Exercises on Elderly's Depression Rate. Iran J Health Educ Health Promot. 2013;1(3):23-32.

28. Xu Y, Ku B.S, Yao H.Y, Lin Y.H, Ma X, Zhang Y.H, et al. The effects of curcumin on depressive-like behaviors in mice. Eur J Pharmacol. 2005;518:40-6.

29. Panahi Y, Hosseini MS, Khalili N, Naimi E, Majeed M, Sahebkar A. Antioxidant and anti-inflammatory effects of curcuminoid-piperine combination in subjects with metabolic syndrome: a randomized controlled trial and an updated meta-analysis. Clin Nutr. 2015;34(6):1101-1108. doi: 10.1016/j.clnu.2014.12.019.

30. Andrew H. Miller, Vladimir Maletic, Charles L. Raison. Inflammation and Its Discontents: The Role of Cytokines in the Pathophysiology of Major Depression. Biol Psychiatry. 2009;65(9):732-41.

31. Schottelius AJ, Mayo MW, Sartor RB, Baldwin AS, Jr. Interleukin-10 signaling blocks inhibitor of kappaB kinase activity and nuclear factor kappaB DNA binding. J Biol Chem. 1999;274(45):3186874.

32. Glaser R, Robles TF, Sheridan J, Malarkey WB, Kiecolt-Glaser JK. Mild depressive symptoms are associated with amplified and prolonged inflammatory responses after influenza virus vaccination in older adults. Arch Gen Psychiatry. 2003;60:1009-14. 
33. Xu Y.X, Pindolia K.R, Janakiraman N, Chapman R.A, Gautam S.C. Curcumin inhibits IL-1 alpha and TNF-alpha induction of AP-1 and NK-kB DNA-binding activity in bone marrow stromal cells. Hematopathol Mol Hematol. 1998;11:49-62.

\section{Tables}

Table 1. The anthropometric indices, body composition of subjects before and after the study 


\begin{tabular}{|c|c|c|c|c|}
\hline \multirow[t]{2}{*}{ Variable } & \multirow[t]{2}{*}{ group } & pre-test & Post-test & P-value \\
\hline & & Mean \pm SD & Mean \pm SD & Between-group \\
\hline \multirow[t]{4}{*}{ Weight $(\mathrm{kg})$} & Training & $43.06 \pm 04.80$ & $04.04 \pm 83.70$ & \multirow{4}{*}{$0.004^{e}$} \\
\hline & Training+ Supplement & $41.04 \pm 13.81$ & $12.06 \pm 68.68$ & \\
\hline & Supplement & $21.07 \pm 71.80$ & $02.03 \pm 75.69$ & \\
\hline & Control & $73.07 \pm 08.79$ & $68.09 \pm 88.80$ & \\
\hline \multirow[t]{4}{*}{$\mathrm{BMI}(\mathrm{kg} / \mathrm{m} 2)$} & Training & $54.04 \pm 63.33$ & $62.03 \pm 43.27$ & \multirow[t]{4}{*}{$0.008^{e}$} \\
\hline & Training+ Supplement & $61.05 \pm 69.34$ & $77.04 \pm 73.26$ & \\
\hline & Supplement & $63.07 \pm 56.33$ & $73.05 \pm 24.27$ & \\
\hline & Control & $38.05 \pm 49.34$ & $35.08 \pm 32.34$ & \\
\hline \multirow[t]{4}{*}{ WH R } & Training & $.3205 \pm 78.104$ & $.6103 \pm 52.93$ & \multirow[t]{4}{*}{$0.006^{\mathrm{e}}$} \\
\hline & Training+ Supplement & $102.56 \pm 06.43$ & $92.63 \pm 03.67$ & \\
\hline & Supplement & $103.42 \pm 05.56$ & $93.63 \pm 02.43$ & \\
\hline & Control & $84.04 \pm 98.103$ & $75.05 \pm 21.104$ & \\
\hline \multirow[t]{4}{*}{$\mathrm{SBP}(\mathrm{mmHg})$} & Training & $.3207 \pm 37.158$ & $.713 \pm 43.121$ & \multirow[t]{4}{*}{$0.007^{e}$} \\
\hline & Training+ Supplement & $.06 .61 \pm 37.160$ & $2.99 \pm 43.120$ & \\
\hline & Supplement & $06.76 \pm 36.157$ & $2.87 \pm 43.122$ & \\
\hline & Control & $63.05 \pm 26.161$ & $58.07 \pm 87.159$ & \\
\hline \multirow[t]{4}{*}{ Triglyceride (mg/dl) } & Training & $34.73 \pm 32.256$ & $142.82 \pm 11.89$ & \multirow[t]{4}{*}{0.008} \\
\hline & Training+ Supplement & $53.45 \pm 08.264$ & $141.98 \pm 12.11$ & \\
\hline & Supplement & $34.74 \pm 32.256$ & $144.54 \pm 12.63$ & \\
\hline & Control & $53.45 \pm 08.264$ & $74.68 \pm 87.260$ & \\
\hline \multirow[t]{4}{*}{$\mathrm{HDL}(\mathrm{mg} / \mathrm{dl})$} & Training & $45.12 \pm 36.41$ & $82.04 \pm 47.53$ & \multirow[t]{4}{*}{0.001} \\
\hline & Training+ Supplement & $41.86 \pm 11.56$ & $54.56 \pm 09.56$ & \\
\hline & Supplement & $42.34 \pm 10.37$ & $54.89 \pm 08.66$ & \\
\hline & Control & $46.04 \pm 23.45$ & $84.06 \pm 64.46$ & \\
\hline \multirow[t]{3}{*}{ Glucose (mg/dl) } & Training & $65.32 \pm 84.182$ & $.7310 \pm 63.115$ & \multirow[t]{3}{*}{0.000} \\
\hline & Training+ Supplement & $185.98 \pm 32.87$ & $114.65 \pm 11.01$ & \\
\hline & Supplement & $184.54 \pm 31.43$ & $113.65 \pm 12.06$ & \\
\hline
\end{tabular}


BMI, Body Mass Index; WHR, waist hip ratio, SBP; Systolic Blood Pressure; HDL, High-density lipoprotein Table 2. Comparison of IL-1 $\beta$, CRP before and after training

\begin{tabular}{|c|c|c|c|c|c|c|}
\hline \multicolumn{7}{|l|}{ Groups } \\
\hline Variable & Stages & Control & $\begin{array}{l}\text { Training+ } \\
\text { Supplement }\end{array}$ & Supplement & Training & $\begin{array}{l}\text { P } \\
\text { (Between } \\
\text { Group ) }\end{array}$ \\
\hline \multirow[t]{5}{*}{$\begin{array}{l}\text { IL1 } \beta \\
(\mathrm{pg} / \mathrm{ml})\end{array}$} & $\begin{array}{l}\text { Pre } \\
\text { test }\end{array}$ & $4.87 \pm 1.45$ & $4.99 \pm 1.33$ & $4.77 \pm 1.89$ & $4.80 \pm 1.45$ & \multirow[t]{2}{*}{$\star 0.002$} \\
\hline & $\begin{array}{l}\text { Post } \\
\text { tetst }\end{array}$ & $4.82 \pm 1.36$ & $2.33 \pm 1.27$ & $2.99 \pm 1.34$ & $2.67 \pm 1.51$ & \\
\hline & $P$ & 0.9 & $\star 0.002$ & $\star 0.05$ & $\star 0.003$ & \\
\hline & (within & & & & & \\
\hline & group) & & & & & \\
\hline \multirow[t]{5}{*}{$\begin{array}{l}\mathrm{NO} \\
(\mathrm{ng} / \mathrm{ml})\end{array}$} & $\begin{array}{l}\text { Pre } \\
\text { test }\end{array}$ & $0.108 \pm 0.095$ & $0.120 \pm 0.03$ & $0.125 \pm 0.074$ & $0.120 \pm 0.023$ & \multirow[t]{2}{*}{$\star 0.002$} \\
\hline & $\begin{array}{l}\text { Post } \\
\text { tetst }\end{array}$ & $0.111 \pm 0.090$ & $0.151 \pm 0.019$ & $0.147 \pm 0.026$ & $0.146 \pm 0.084$ & \\
\hline & $\mathrm{P}$ & 0.4 & $\star 0.002$ & $\star 0.002$ & $* 0.002$ & \\
\hline & (within & & & & & \\
\hline & group) & & & & & \\
\hline \multirow[t]{3}{*}{ Deprestion } & $\begin{array}{l}\text { Pre } \\
\text { test }\end{array}$ & $22.87 \pm 5.23$ & $22.65 \pm 4.33$ & $20.46 \pm 4.45$ & $21.98 \pm 5.56$ & \multirow[t]{3}{*}{$* 0.001$} \\
\hline & $\begin{array}{l}\text { Post } \\
\text { tetst }\end{array}$ & $21.93 \pm 5.15$ & $11.76 \pm 2.11$ & $10.11 \pm 2.17$ & $12.57 \pm 5.02$ & \\
\hline & & 0.09 & $* 0.001$ & $* 0.001$ & $\star 0.001$ & \\
\hline
\end{tabular}

IL1ß,Interleukin 1 beta; NO, Nitric Oxide 